\title{
Louise Bienvenue, Ollivier Hubert et Christine Hudon \\ Le collège classique pour garçons. Études historiques sur une institution québécoise disparue
}

Montréal : Fides, 2014. 418 pages.

\section{Mathilde Cambron-Goulet}

Université du Québec à Montréal

Le collège classique pour garçons est un recueil d'études croisées sur un lieu - le collège classique - à partir d'une perspective commune originale — la fabrication de la masculinité. Quoiqu'il ne s'agisse pas d'une discussion sur l'école, les auteurs abordent des thèmes tels que le curriculum scolaire des collèges (chapitres 1 et 3 ), la manière dont celui-ci constitue un lieu de production d'inégalités (chapitre 4) et de construction d'une conscience de classe (chapitre 14), ainsi que les caractéristiques des différents acteurs du milieu scolaire, qu'il s'agisse des enseignants (chapitre 7), des cohortes étudiantes (chapitre 2) ou des "petites sœurs " qui assurent le travail domestique dans les collèges (chapitre 8). Louise Bienvenue, Ollivier Hubert et Christine Hudon parviennent ainsi à décrire les processus de construction du masculin au collège et par là, à dépasser les dichotomies souvent attachées à un objet d'étude devenu mythe grâce à l'étude minutieuse de sources fort diversifiées (archives, traités pédagogiques, journaux, publicités, romans, entrevues).

D'abord, Christine Hudon souligne que le collège classique ne se distingue pas nécessairement par un curriculum axé sur les humanités; vers 1830, le programme du collège de Sainte-Anne-de-la-Pocatière adapte le Ratio studiorum des Jésuites aux réalités politiques et sociales, et jusque vers 1940, le cours commercial prédomine dans ce collège (chapitre 1). Les cohortes étudiantes, rappelle Ollivier Hubert, ont plutôt en commun un parcours variable qu'un parcours classique (chapitre 2). En écho, Louise Bienvenue, revisitant l'opposition entre la " tradition " (enseignement des humanités) et la "modernité " (cours commerciaux et anglais), montre que 
c'est un effort de distinction par rapport à la concurrence qui amène le collège à se " classiciser ", malgré un mouvement en faveur du cours commercial et de l'anglais, défendu notamment par Lionel Groulx (chapitre 3). De fait, tous les collégiens ne développent pas la maîtrise du latin qui leur permettrait d'accéder à l'université, car le réseau scolaire opère une double ségrégation des jeunes hommes sur la base de la fortune des parents et du latin (O. Hubert, chapitre 4). Les humanités sont reléguées au second plan jusque dans les prospectus des collèges (C. Hudon, chapitre 13) : loin de reposer sur la promotion de la culture latine et avant de s'appuyer au début $\mathrm{du} \mathrm{XX}^{\mathrm{e}}$ siècle sur des principes disciplinaires et religieux, ceux-ci mettaient de l'avant au $\mathrm{XIX}^{\mathrm{e}}$ siècle un environnement romantique ou hygiénique (qui est l'occasion de savoureuses envolées lyriques, 331-332). La nostalgie actuelle d'un cours classique mettant de l'avant la culture classique, mentionnée dans l'introduction et étudiée dans l'épilogue, en prend pour son rhume.

La dichotomie entre un collège clos, homosocial, et le monde extérieur, traverse l'ouvrage. Louise Bienvenue et Christine Hudon illustrent la distance qui sépare le collège de la vie extérieure dans des articles sur l'entre-soi de genre (chapitre 10) et l'omniprésence des femmes et de la sexualité dans les collèges (chapitre 11). Dans un milieu presque exclusivement masculin qui néglige l'éducation des hommes à la vie conjugale, comme le déplorent dans les années 1940 et 1950 épouses, psychologues et anciens collégiens, seule l'amitié est l'occasion d'emprunter au discours amoureux et de s'y préparer. Cela dit, les collèges n'ont pas toujours été des lieux coupés du monde, dans la mesure où les collèges visent la formation d'une relève cléricale mais œuvrent à catholiciser la société (O. Hubert, chapitre 5), aussi la majorité des collégiens ne sont pas les pensionnaires-latinistes pour lesquels les règlements du collège sont conçus (O. Hubert, chapitre 6). Ces règlements ne sont pas toujours respectés, ni par les étudiants, ni par les enseignants qui se font parfois leurs complices (O. Hubert, chapitre 6, C. Hudon et L. Bienvenue, chapitres 7 et 9). Le chapitre 9, qui identifie diverses manières illicites d'acquérir une identité masculine dans un milieu jugé peu viril, permet de redécouvrir des transgressions : les lectures interdites, la grève du zèle à la messe et le trafic de billets de confessions sont décrits à l'aide d'exemples évocateurs, ainsi cet éducateur tâchant d'expurger les pièces de Racine de ses rôles féminins laisse entrevoir un milieu dont le caractère homosocial est parfois artificiellement amplifié (240).

Enfin, les auteurs montrent que comme milieu homosocial, le collège joue un rôle central dans la construction de l'identité de genre. Les chercheurs s'appuient pour ce faire sur divers modèles traditionnels, réformistes et modernes de masculinité (chapitre 15), définis par rapport aux enseignants (chapitre 7), à la famille (chapitre 15) ou à une classe sociale (chapitres 2, 4, 14); sur la zone de tolérance des collèges vis-à-vis des infractions mineures (chapitres 5, 6, 7 et 9) ; sur la place des femmes dans le milieu scolaire (chapitres 8 et 11) ; voire sur des discours théologiques qui viennent à la rescousse des sports au collège (chapitre 12) ou de l'éducation sexuelle (chapitre 11). Le tout fait en outre apparaître un genre féminin tantôt cantonné à un rôle conventionnel, alors que les femmes ne peuvent ni être héroïnes ni auteures (chapitre 15), tantôt tirant parti de ce rôle : quoique les communautés féminines qui 
assurent le travail domestique dans les collèges œuvrent dans des conditions difficiles, la difficulté des directeurs de collège à recruter des hommes de ménage leur donne un certain pouvoir de négociation (L. Bienvenue et G. Laperrière, chapitre 8).

L'ouvrage constitue un ensemble cohérent d'une richesse remarquable, qui évite avec doigté les écueils liés au traitement d'un "lieu de mémoire " (389), voire d'un mythe dont l'héritage est à la fois glorifié et rejeté, ou au traitement de questions délicates, telles que l'attirance sexuelle dans un contexte scolaire (chapitres 7 et 10). Quoiqu'on puisse regretter l'absence d'un index thématique et de références croisées entre les articles, tant la lecture aiguisée des sources que la discussion approfondie de l'historiographie font de cet ouvrage un travail d'une rigueur exemplaire que contempteurs et thuriféraires du collège classique auront plaisir à lire, pour peu qu'ils veuillent renouveler le regard qu'ils portent sur cette institution. 